\title{
Application of the SECI Model Using Web Tools to Support Diabetes Self-Management and Education in the Kingdom of Saudi Arabia
}

\author{
Saleh Almuayqil1,2, Anthony S. Atkins' ${ }^{2}$, Bernadette Sharp² \\ ${ }^{1}$ College of Computer Sciences and Information, Aljouf University, Sakaka, KSA \\ ${ }^{2}$ School of Computing and Digital Technologies, Staffordshire University, Stoke-on-Trent, UK \\ Email: saleh.naif@ju.edu.sa
}

How to cite this paper: Almuayqil, S., Atkins, A.S. and Sharp, B. (2017) Application of the SECI Model Using Web Tools to Support Diabetes Self-Management and Education in the Kingdom of Saudi Arabia. Intelligent Information Management, 9, 156-176.

https://doi.org/10.4236/iim.2017.95008

Received: July 17, 2017

Accepted: August 20, 2017

Published: August 23, 2017

Copyright $\odot 2017$ by authors and Scientific Research Publishing Inc. This work is licensed under the Creative Commons Attribution International License (CC BY 4.0).

http://creativecommons.org/licenses/by/4.0/ (c) (i) Open Access

\begin{abstract}
The area of knowledge management, the SECI mode in particular, has great value in terms of enriching patients' knowledge about their diseases and its complications. Despite its effectiveness, the application of knowledge management in the healthcare sector in the Kingdom of Saudi Arabia seems deficient, leading to insufficient practice of self-management and education of different prevalent diseases in the Kingdom. Moreover, the SECI model seems to be only focusing in the conversion of human knowledge and ignore knowledge stored in databases and other technological means. In this paper, we propose a framework to support diabetic patients and healthcare professionals in the Kingdom of Saudi Arabia to self-manage their disease. Data mining and the SECI model can provide effective mechanisms to support people with diabetes mellitus. The area of data mining has long been utilised to discover useful knowledge whereas the SECI model facilitates knowledge conversion between tacit and explicit knowledge among different individuals. The paper also investigates the possibilities of applying the model in the web environment and reviews the tools available in the internet that can apply the four modes of the SECI model. This review helps in providing a new median for knowledge management by addressing several cultural obstacles in the Kingdom.
\end{abstract}

\section{Keywords}

SECI Model, Framework, Saudi Arabia, Diabetes Mellitus

\section{Introduction}

The Kingdom of Saudi Arabia (KSA) has been associated for increased preva- 
lence of number of diseases including diabetes mellitus. The prevalence of these diseases in KSA requires daily monitoring and continuous care to prevent its complications. An effective way to manage these diseases is the application of self-management and education strategies. The term self-management in the medical domain is used to emphasize the patients' role to control their diseases. The term has been used to enable individuals to deal with their chronic illnesses, and its resulted complications, by increasing their knowledge, skills and confidence [1]. Self-management also empowers people with different diseases to manage their negative emotions and maintain life roles. It has been applied globally and has proved its usefulness to a number of diseases including different chronic conditions. However, its application in KSA is deficient and doesn't meet the required effort to control these diseases.

The application of self-manage and education in healthcare can be supported by Knowledge Management (KM). KM is a dynamic process of capturing, storing, sharing and creating knowledge [2]. KM facilitates sharing of knowledge among different organizations and individuals through communications and offers useful means for creating and disseminating knowledge [3]. Despites its advantages, $\mathrm{KM}$ in KSA face several factors related to national and organisational aspects that hinder its implementation and limit its practices in different domains including healthcare. Lack of KM practice in KSA limits application of several activities associated with the idea of self-management and education to support patients of different diseases in the Kingdom.

Nonaka and Takeuchi [4] proposed the knowledge creation model that facilitates interaction of tacit and explicit knowledge by applying the four conversion modes Socialization, Externalisation, Combination and Internalisation (SECI). The SECI model is well-known theory in the area of KM and has been successfully applied to manage knowledge in a variety of domains including healthcare. The SECI model can be utilised to integrate KM and Knowledge Discovery (KD) to support patients and healthcare professionals in KSA in managing several chronic diseases such as diabetes mellitus. Such integration applies KD to elicit tacit complications encountered by diabetic individuals whereas KM helps overcome these complications through the transfer of useful guidelines and best practices among patients with diabetes and healthcare professionals.

DM implementation in healthcare is well documented in the literature [5] [6]. However, the utilisation of the SECI model in the healthcare domain is still deficient. The SECI model explores the interrelationship between the two different types of knowledge, tacit and explicit, and facilitates the conversion between them. Such a process is necessary to benefit from knowledge availability in the healthcare domain [7]. The SECI model has been utilised in different domains for the purpose of knowledge creation and dissemination. Several studies have shown successful applications of the knowledge creation model successfully. For example, the SECI model was proposed to be applied in a library to build a system for knowledge sharing [8]. In another study, the SECI model was utilised in 
the field of software development in order to assist stakeholders in the process of elicitation, specification and validation of software requirements [9]. Additionally, another study examined the SECI model utilisation in the manufacturing sector and proved its successful utilisation [10]. Nevertheless, the successful implementation in different domains requires similar application within the healthcare sector in KSA, which is seen to have slower KM practice than other healthcare sectors in developed countries [11] [12] [13].

Although the SECI model is recognized as valuable approach for knowledge creation, the concentration of the model tends to primary focus on social interactions to share human knowledge; yet there is a rich knowledge embedded in databases and documents to be mined and shared using this model, making use of many available software tools. In this paper, the focus is how the SECI model can disseminate such knowledge using a set of web tools. Internet technologies are widely utilised in order to self-manage illnesses and learn from other patients' experiences [14]. Therefore, the application of the SECI to support effective interaction between patients and healthcare professionals can be achieved through the utilisation of many related web tools. Such an approach can assist promote the sharing of guidelines and best practices among patients and healthcare professionals to manage diseases and improve their wellbeing.

This paper presents an integrated framework of KM and KD to support he community of diabetes mellitus in KSA. The framework utilises the SECI model to fulfil two main issues in the context of KSA. Firstly, the lack of KM practices in healthcare. Secondly, the lack of diabetes self-management and education initiatives. The paper is organised as follows: Section 2 highlights diabetes mellitus in KSA. Section 3 discusses the application of KM in the healthcare domain in KSA. Section 4 defines the SECI model. Section 5 introduces our integrated framework. Section 6 provides a literature review of the web tools that can be applied to the SECI model. Section 7 draws the conclusion of this paper and highlights areas of future work.

\section{Diabetes Mellitus in the Kingdom of Saudi Arabia}

Diabetes mellitus is a group of metabolic disorders known by hyperglycaemia which is caused by deficient insulin secretion and/or insulin actions [15]. People in different ageing, gender and in developing and even developed countries are being diagnosed with diabetes and the prevalence of diabetes mellitus is expected to increase internationally to 592 million in 2035 [16].

KSA, like other developing countries, has a considerable number of patients who are suffering from different kind of chronic diseases. One of those diseases is diabetes mellitus, which has high percentage of appearance among citizens in almost every part of the Kingdom. According to [17], KSA is in the third place among the top 10 countries for diabetes prevalence. In 2010, the prevalence percentage for diabetes mellitus in KSA was $16.8 \%$ among adults in the ages of 20 79 years old. This percentage is expected to rise in 2030 to reach $18.9 \%$ [18]. The 
number of outpatient visits made by diabetic individuals to Primary Healthcare Centres (PHCs) is 1.8 million out of 29.3 million overall outpatient visits to PHCs in KSA. This number of diabetic people's visits represents $6.44 \%$ of the overall number of visits to all PHCs in KSA and put diabetic people in third place in the scale of all outpatient visits in the Kingdom [19].

Official reports from the $\mathrm{MOH}$ in KSA show increasing numbers of visits to public hospitals over the years. The number of visits to $\mathrm{MOH}$ hospitals due to diabetes mellitus was over 434 thousands in 2011 [19]. The number has increased through 4 years to exceed 477 thousand visits to MOH hospitals in 2014 [20].

In a recent statistical study, the International Diabetes Federation revealed that KSA has more than 3.8 million cases of diabetes mellitus in 2014 and the prevalence of this disease among individuals represents $20.5 \%$ [21]. Those diabetic people are not limited to a particular age. In 2015, KSA is in the list of top countries in terms of the prevalence of type 1 diabetes among children under 14 years old with 16,100 cases [22]. In the same year, the prevalence of diabetes mellitus among people from 20 to 79 years old is $20 \%$. Diabetic people in KSA suffer from various complications of diabetes. However, the most serious complication encountered by diabetics in KSA is mortality. According to the Centers for Disease Control and Prevention, diabetes mellitus is considered the first cause of death in KSA among all other diseases in 2013 [23].

Beside many complications which could result from this disease, diabetic individuals also face other difficulties and issues related to controlling the disease and its consequences through different lifestyle activities. These issues are known by the non-health related complications of diabetes mellitus. Péres et al. [24] reported some of those challenges people with diabetes mellitus can have in their daily life including difficulties controlling impulses related to eating habits, doubts in the correct way of using medications, refusal to take insulin because of concerns regarding dose preciseness and doubts regarding time schedules for antidiabetics. Blonde [25], in addition, indicated patient deficiency in adherence to lifestyle measures and pharmacologic therapies as one of the most common reasons cited for failure to achieve glycaemic goals. Patients of diabetes face problems in identifying medications and understanding prescriptions especially when patients asked to change medication. Most patients could not remember their blood glucose and blood pressure target. According to Onwudiwe et al. [26], the lack of knowledge of desired blood glucose level is the major barrier of diabetes self-management. Another study suggested that appropriate knowledge about diabetes mellitus is essential to ensure patients' adherence to medication [27]. The study found that diabetic individuals expressed their concerns regarding the adverse effects of multiple anti-diabetes medications. In addition, participants in the study believe that diabetes medications can be harmful, which suggests assessment of patients' knowledge and education about diabetes mellitus prior to medication intake to ensure adherence of patients to medications. 
In addition to the above complications, diabetic patients in Muslim countries face difficulties related to controlling diabetes during the Holy month of Ramadan. Since people in Muslim countries fast every day of the month of Ramadan from sunrise until sunset, Muslims who have diabetes mellitus might be at risk of considerable fluctuations in the blood glucose level [28]. Muslims in Ramadan change their eating routines, which includes changing the types and timing of almost all meals during this month. This can affect fasting people with diabetes mellitus in terms of identifying the appropriate diet, medication time and amount [29].

Diabetes mellitus has wider attention in the area of self-management and education from variety of studies which have demonstrated such programs in different countries [25] [30] [31]. However, the implementation of diabetes selfmanagement and education in KSA is still deficient especially when considering the vast prevalence of the disease in the Kingdom.

\section{Knowledge Management in Healthcare in the Kingdom of Saudi Arabia}

The healthcare domain utilises tacit and explicit knowledge in different ways. Explicit knowledge is communicable in systematic language whereas tacit knowledge is obtained through experience and cannot be easily articulated [4]. Researchers have demonstrated the importance of the two types of knowledge in different studies. Tacit knowledge is valuable for many healthcare practitioners in addressing a medical problem [32]. Tacit knowledge is also beneficial in clinical care as it is the basis for decision-making even though these decisions guidelines are made available in an explicit form [33]. The value of tacit knowledge in this context resides in capturing such a form of knowledge which can contribute to the performance and training of practice, typically based on experience [34]. More precisely, utilisation of tacit knowledge in healthcare has more connotations to healthcare delivery by promoting or rejecting of explicit medical expertise [35]. Moreover, the capture of tacit knowledge, and its transformation into explicit, seems to benefit the performance not only for clinicians, but also for hospital leadership and other individuals in different departments in the hospital [36].

Explicit knowledge is also useful to capture critical issues in the healthcare sector. Knowledge is the driving force for enhancing practice management [37]. Therefore, making this knowledge explicit can make healthcare organisations better equipped to deal with constraints such as increased cost and the pressure for delivering high quality and effective care. In addition, explicit knowledge is valuable for the practice of evidence based medicine, which is based on research evidence, clinical expertise and patients' preferences and values for the purpose to achieve best medical decision-making [37].

The advantages of tacit and explicit knowledge as well as the case of evidence based medicine raise the importance of utilising a conversion mechanism for the 
two types of knowledge in healthcare. Evidence based medicine is primarily based on the explicit evidence from research. However, it is important to consider the tacit knowledge which represents both the clinical expertise of medical staffs and the patients' preferences. According to Wickramasinghe and Davison [37], the tacit knowledge of medical expertise and patients' preferences are not utilised sufficiently. More importantly, it is almost impossible for healthcare professionals to rely on solely tacit knowledge in supporting their medical decisions and practices. Wickramasinghe and Davison also stated that ignoring the tacit knowledge of healthcare professionals, which is gained from daily practices, might not be the solution as that tacit knowledge can provide great significance to the healthcare system. Therefore, the best solution to tackle this problem is to adopt a knowledge conversion theory that can convert tacit and explicit knowledge to support relevant activities in the healthcare domain.

Conversion between tacit and explicit knowledge not only benefits healthcare professionals and medical staff in healthcare, it can also benefit patients by facilitating sharing and converting tacit and explicit knowledge about their diseases, and its associated aspects such as diagnosis and medication. The knowledge which patients have about their diseases is the first step in their treatment journey; it can affect their lives as it can determine their activities and lifestyle [11]. This can contribute to their control and management of their diseases. Application of KM in the healthcare sector can be effective in sharing knowledge to educate patients about their diseases and how to manage them efficiently.

The education influence, offered to patients and other stakeholders, is well documented in developed countries; which plays significant role in empowering patients and enriching their knowledge about their diseases. However, such an influence is still deficient in Saudi Arabian healthcare sector [11]. More precisely, small number of large healthcare organisations in the Kingdom has documented education efforts to support their patients about their diseases and medication. For example, Al-Ghamdi et al. [38] assessed a medication counselling program provided for discharged patents from a hospital in Saudi Arabia to avoid adverse drug events. The study showed that the knowledge and education effort provided for patients prevented them from adverse drug events. In another study conducted in another major hospital in the Kingdom, the education and counselling program increased the medical knowledge of patients in relation to their recognition of medication and its side effects [39].

Nevertheless, the majority of healthcare organisations in KSA lack an effort in relations to educate their patients about the different diseases they suffer from. The reason beyond this deficiency can be linked to poor KM practice due to a number of factors. The literature indicates these factors are uniquely derived from the national and organisational cultures in most organisations in the Arabic, Middle Eastern and Saudi Arabian Context. These factors are highlighted as follows:

- Trust is a strong factor that influences the sharing of knowledge among 
members in an organisation [40] [41]. Trust creates the atmosphere necessary for engaging with others especially when the regulations and rules insufficiently ensure their perceived behaviour [40]. The presence of trust among members is the core factor towards successful knowledge sharing among them [42]. In addition, trust will enable the availability of knowledge to partners and colleagues even though the individuals who own that knowledge prefer to keep it for themselves [43].

- Openness to change is important for promoting, managing and sharing knowledge [28] [29] [44] [45]. Openness is defined by Bradley [46] as improving performance or obtaining high absorptive capacity through recognising and responding to the needs for change.

- Team-work is having the members of any organisation to work as a team. Team-working facilitates the success of knowledge sharing process by fostering members to contact and eventually learn from each other's [47]. Previous studies have shown the positive effect of working as a team and emphasised that it improve and enable knowledge sharing and management among members [48] [49]. However, organisations in Saudi Arabia provide limited opportunities to engage members from different departments to engage in teamwork practices [50].

- Communication and interaction between members inside and outside the organisation is essential for sharing knowledge [41]. One of the barriers of KM practices in Saudi Arabian context is the lack of effective organisational communication [51]. Glisby and Holden [52] suggested that cooperative work environment is necessary for Knowledge Sharing (KS). Individuals who experience competition in organisation feel the stress leading to discouraging KS in their organisation [53].

- The structure of an organisation is another factor in the organisational culture that influences KM practices in different organisations [54]. Middle Eastern cultures are considered to have high level of bureaucracy which can hinder KS activities within any organisation [55].

- Some organisations have insufficient KS practice due to the high power distance barrier, which is an attribute driven by the tradition [56] [57]. In high power distance cultures, managers normally control their employees, and workers in the workplace are reluctant to speak with their managers openly. Employees in those cultures fear the communication with their managers because of the high level of inequality in power distribution among individuals. Consequently, sharing of knowledge is negatively influenced. Countries in the Arab world are regarded as high power distance cultures and this will affect externalisation and internalisation of knowledge [53].

\section{About the SECI Model}

As Nonaka and Takeuchi explain in their paper that though organisations actually create and manage knowledge dynamically, there is little understanding of 
how to capture it and share it. They propose a model of knowledge creation consisting of three elements: the SECI (Socialisation, Externalisation, Combination, and Internalisation) process, knowledge creation through the conversion of tacit and explicit knowledge, the shared context for knowledge creation, and knowledge assets [58]. The knowledge creation process is a spiral which grows out of these three elements and can lead to dialectical thinking. Using existing knowledge assets, organisations create new knowledge through the SECI process that takes place in the share context; new created knowledge becomes in turn the basis for a new spiral of knowledge creation. They identify two types of knowledge, explicit and tacit, which are important to share and convert them in order to create knowledge. The four SECI conversion modes are explained below and illustrated in Figure 1.

Socialisation. Socialisation is the first mode in the SECI model and involves social interaction to enable the conversion of tacit knowledge from one to another without use of language [59]. Observation, imitation and brainstorming are useful tools to perform such a conversion.

Externalisation. The second mode of the SECI model is externalisation which enables the conversion from tacit to explicit knowledge [59]. It translates tacit knowledge to forms to be understandable and interpretable by other users via interaction, namely dialogues and informal meetings. The new produced explicit knowledge can then can be codified and recorded through different means such documentation and recording.

Combination. The conversion of explicit knowledge to another explicit knowledge is the third mode of the SECI model [59]. This process can be performed through combining different explicit knowledge and reconfiguring a new form of explicit knowledge. Formal meetings in organisations are considered as a very assistive combination tool [4].

Internalisation. Internalisation is the mode where explicit knowledge is converted to tacit knowledge and become part of an individual's brain [4]. The im-

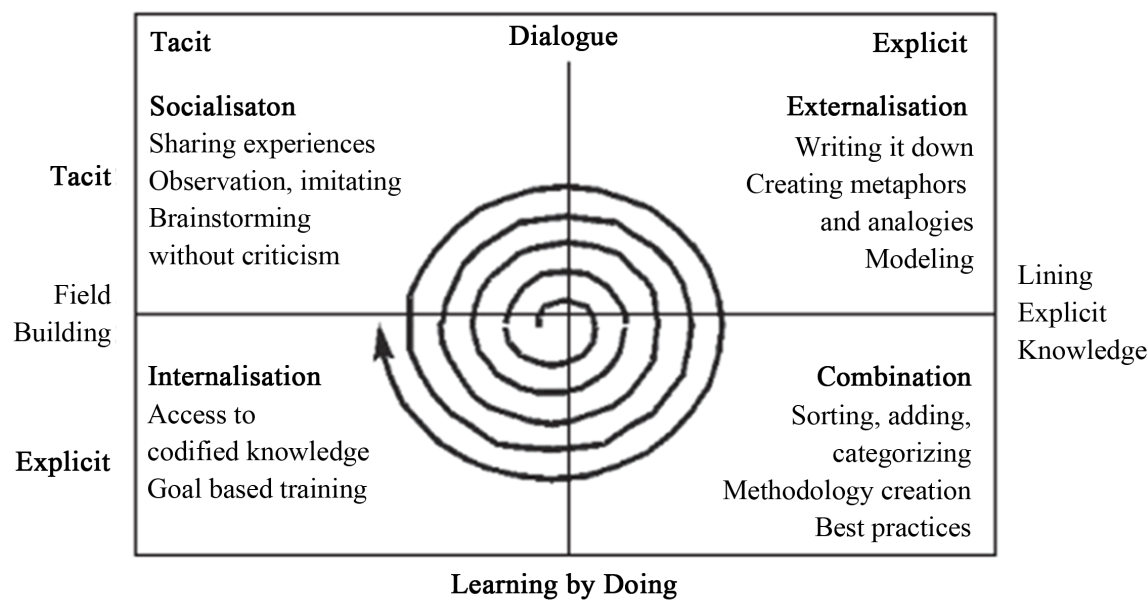

Figure 1. The SECI model [1]. 
plementation of internalisation involves "learning by doing" and individuals who successfully internalise tacit knowledge will eventually acquire "know how" skills.

An important factor that must be considered when discussing the SECI model is the concept of " $\mathrm{Ba}$ ". $\mathrm{Ba}$ is a platform where each conversion mode of the SECI model takes place. There are four types of Ba defined by [60]. Ba is the space where individuals share their experiences, feelings and emotions. This type of $\mathrm{Ba}$ offers the space where socialisation can be accomplished through physical and face-to-face interactions. Interacting $\mathrm{Ba}$ is the space where mental skills are transformed into explicit form through dialogues. Communication skills and metaphor are essential in this stage of knowledge conversion for successful dissemination of knowledge with respect to its sensitive meanings. Cyber Ba is the third space where combination mode is accomplished through interaction in a virtual world instead of real place and time. The combination mode is performed more efficiently through the utilisation of technology. The fourth type of $\mathrm{Ba}$ is the exercising $\mathrm{Ba}$ which is the space that facilitates internalisation through training and practical learning.

Although the application of KM seen to be hindered due to several factors in KSA, the SECI model offer a solid basis to encourage KM to empower patients' of diabetes mellitus in the Kingdom in terms of self-management and education; the SECI model can be tailored and adapted to meet the cultural constraints associated with the Saudi societies to ensure healthcare organisations. Individuals can survive in the knowledge economy environment and that knowledge can be effectively managed and shared among the diabetic individuals. Solutions have been suggested to support KM practices through the adoption of the SECI model [61]. These solutions provide useful tools that can emphasize human interactions, an essential component of KM in healthcare organisations and other sectors in KSA. Therefore, the current research has applied the SECI model to increase the knowledge of citizens who struggle from diabetes mellitus in KSA to fulfil the gap of deficient KM utilisation in the Saudi Arabian healthcare sector.

\section{The Framework}

The framework is designed in three layers (see Figure 2). The first layer focuses on the barriers related to e-health in KSA and the complications that can be related to different diseases. The framework is applied to the domain of diabetes mellitus in KSA. Therefore, the framework aims to overcome several non-health related complications related to diabetes mellitus. The second layer is related to $\mathrm{KD}$ and DM in particular, where the diabetes mellitus non-health related complications obtained from the first layer are data mined. The third layer is the KM layer where the SECI model is applied to capture these complications and disseminate useful diabetes self-management and provide education guidelines and best practices to diabetic citizens in KSA. 


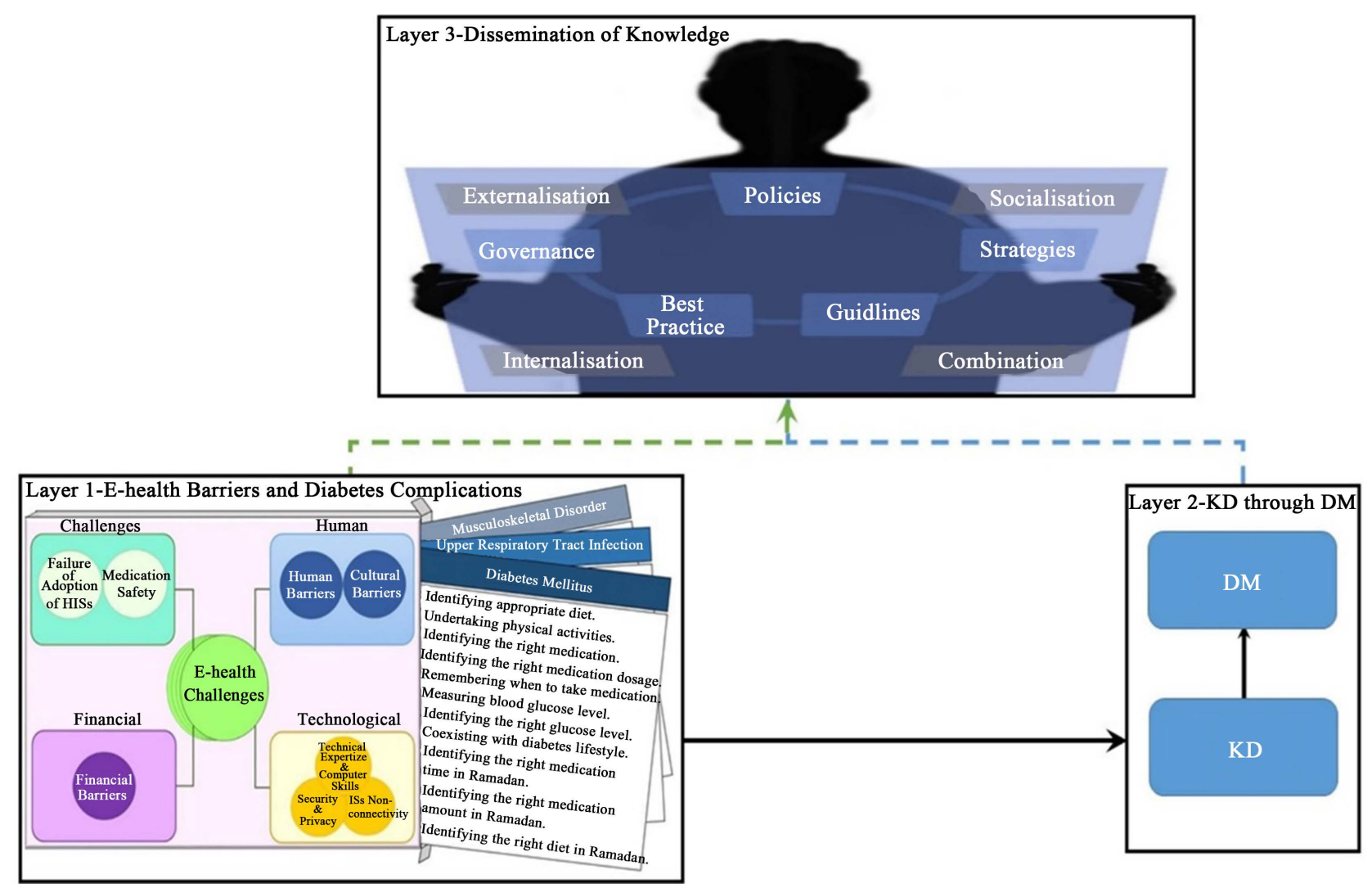

Figure 2. The integrated framework of $\mathrm{KD}$ and $\mathrm{KM}$ for diabetes mellitus in KSA.

\subsection{The First Layer: Issues and Complications}

In this layer, there are two categories of issues, firstly the e-health barriers and secondly the complications related to a particular disease in KSA. The e-health issues are discussed in details in [62], eliciting several e-health issues to the majority of Information and Communication Technologies (ICTs) being adopted in healthcare in KSA. In this paper, the second issue is discussed. The framework is applied to overcome the non-health related complications encountered by diabetic patients in KSA. Diabetes non-health related complications relate to difficulties encountered by diabetes mellitus sufferers in KSA in their daily life. A survey was undertaken to investigate these complications related to lifestyle issues and the medication aspects and profile attributes of diabetic citizens (e.g. age, gender, educational level, etc.). In this survey, we focused on 11 complications, namely diet, physical activities, medication (i.e. dosage intake), blood glucose level, identifying the right glucose level, lifestyle, identifying the right medication time, amount and the appropriate diet in Ramadan (see Figure 3).

\subsection{The Second Layer: Knowledge Discovery and Data Mining}

The aim of this layer is to mine the data acquired from the survey and elicit new knowledge that can link the non-health related complications encountered by diabetic citizens in KSA with the their profile characteristics. Consequently, the 
- Identifying appropriate diet.

- Undertaking physical activities.

- Identifying the right medication.

- Identifying the right medication dosage.

- Remembering when to take medication.

- Measuring blood glucose level.

- Identifying the right glucose level.

- Coexisting with diabetes lifestyle.

- Identifying the right medication time in Ramadan.

- Identifying the right medication amount in Ramadan.

- Identifying the right diet in Ramadan.

Figure 3. Diabetes non-health related complications.

DM method employed is association rule mining, which is appropriate to the structure of our survey which contains nominal attributes and instances.

The findings from the DM study can include different associations among diabetes non-health related complications and the profile information of patients who encounter these complications. For example, the DM study in this layer can link the difficulty of identifying the appropriate diet with diabetic patients who are male, married and have type 1 diabetes.

\subsection{The Third Layer: Knowledge Management}

The third layer in the framework is designed to enable effective provision of useful strategies, guidelines and best practices to citizens relevant to these complications related to different illness. In addition, this layer facilitates the exchange and conversion of tacit and explicit knowledge between patients and healthcare professionals in order to provide useful recommendations that can empower patients to control their diseases. The SECI model is applied in this layer to provide an effective self-management and education tips of diabetes mellitus (see Figure 4).

Policies, strategies, guidelines, best practice and governance are useful recommendations to be shared between stakeholders: patients and healthcare professionals. These aspects provide promising solutions to overcome every nonhealth related complication of diabetes mellitus. Some of these solutions, such as medication intake guidelines, might be in explicit form of knowledge whilst others, such as physical activities best practices, can be tacit. The SECI model enables knowledge creation as the solution presented in one form of knowledge (tacit or explicit) can be transformed to another in order to facilitate effective diabetes self-management for every diabetic citizen in KSA and successful ehealth utilisation in the healthcare sector in the Kingdom. 


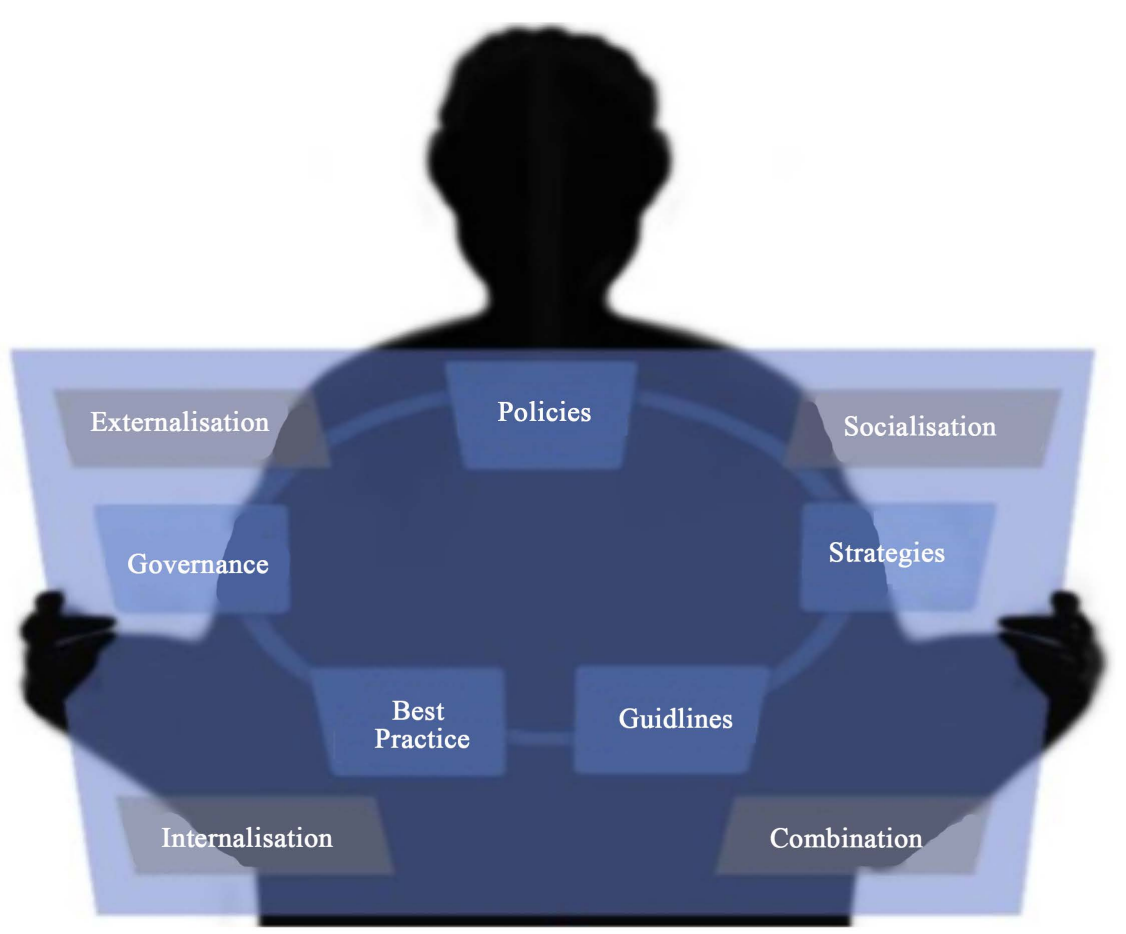

Figure 4. The KM layer.

The first mode in the SECI model, socialization, can overcome the complication of undertaking physical activity as diabetic citizens can socialise with other diabetics who match their diabetes type and profile information such as age, gender and education level. The externalisation mode can be applied to overcome the complication of identifying the right medication dosage through sharing medication guidelines from healthcare professionals to diabetic citizens of a particular diabetes type. The combination mode can overcome the complication of identifying the appropriate medication time in Ramadan through dissemination of different explicit strategies in relation to how to deal with diabetes in the holy month of Ramadan. The internalisation mode can help overcome the complication of identifying the diet appropriate to the health condition through the provision of best practices related to diet by individuals of the same profile information and diabetes type.

\section{Web Tools for the SECI Model}

The above KM layer is supported by a web portal which includes a number of tools to capture the four modes of the SECI model. This portal captures valuable guidelines, strategies and best practices that can help diabetic citizens to control their disease. The portal is named Diabetes Self-Management in the Kingdom of Saudi Arabia (DSMKSA) and is designed with respect to barriers affecting ehealth initiatives in KSA as discussed in [62] (see Figure 5).

Researchers have demonstrated that socialisation, externalisation, combination and internalisation are performed through the internet by various tools 

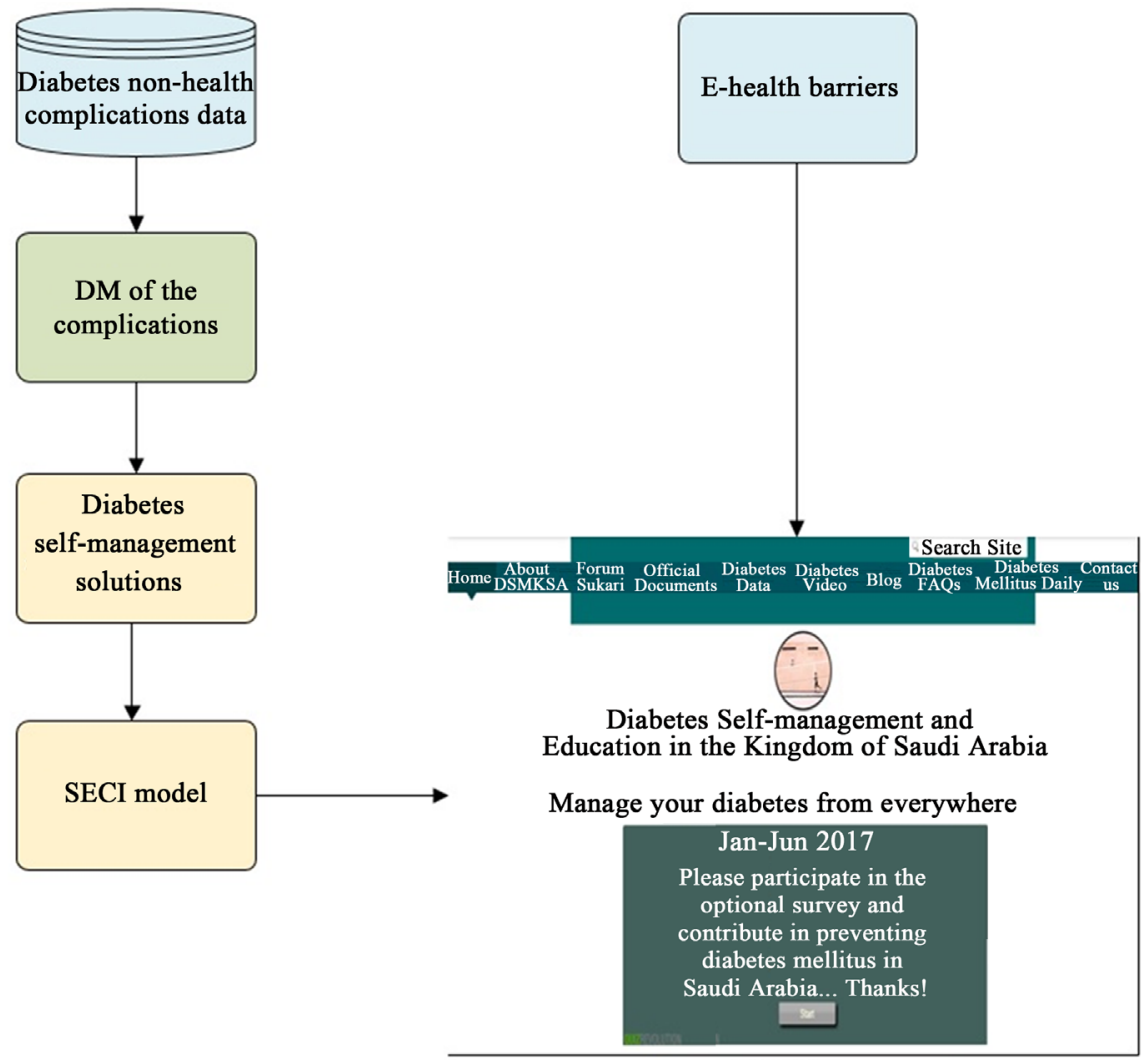

DSMKSA

Figure 5. Embodiment of the KM layer of our framework in a web portal.

provided by the web 2.0 technologies [63] [64]. Web 2.0 is an open source and interactive applications, generated and controlled by the users, which in turn expand their knowledge skills as participants in business and social processes [65]. Web 2.0 enhances the flow of knowledge as it enables the creation of informal networks of users which facilitates the generation, dissemination and editing of knowledge and informational contents. There are a number of web tools, such as blogs, wikis, forums, etc. that can support the four modes of the SECI model. These tools apply the four modes on Nonaka and Takeuchi and address several cultural factors that hinder KM in KSA as outlined below (see Figure 6):

\section{Socialisation:}

1) Forums are a very useful tool to enable socialisation among individuals. Reward incentives can be used for the posts which are highly rated, and will encourage participation [53]. Forums can allow anonymous participation which enables individuals who fear judgments and the loss of face to engage in discussions. This encourages participants' willingness to share their concerns about diet or medication side effects [4].

2) Community of Practice ( $\mathrm{CoP})$ and wikis are useful tools to perform socialisation. According to Ray [53] and Hoadley [66], CoP provided via wikis enables individuals to provide valuable knowledge related to their areas of expertise. Similar to web forums, wikis can enable anonymous participations. This can- 


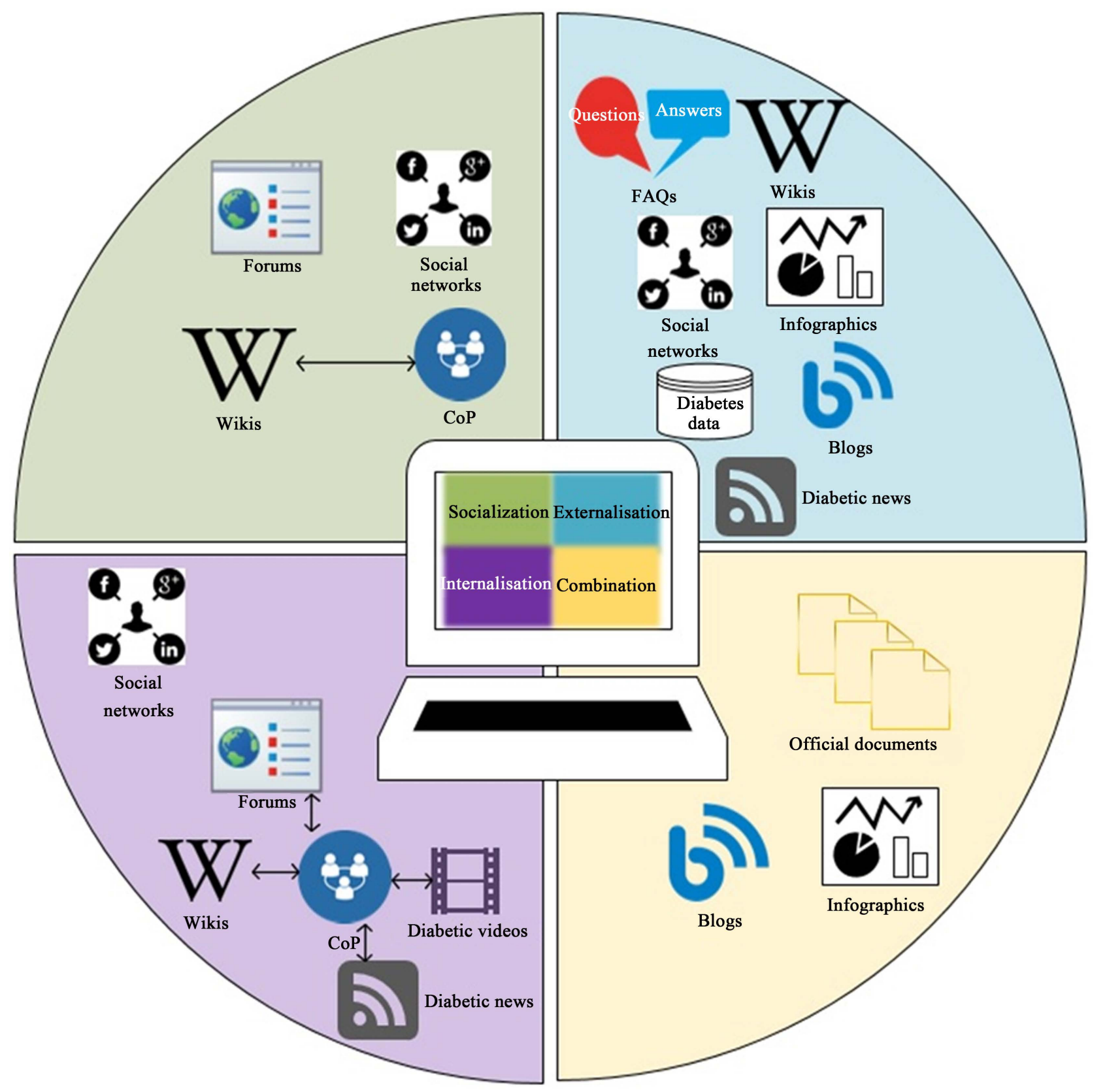

Figure 6. Web tools to apply the SECI model in the internet.

enable KM practices in cultures who are seen as high power distance cultures such as KSA.

3) Social media is another web tool that can enhance socialisation in the internet platform. Social media provides feasible way of sharing tacit knowledge such as expertise, understanding, experience and skills without the utilisation of language. For example, it can disseminate best practices of appropriate exercises. In addition, different social media enable observations, imitation and practice by providing opportunities to individuals to participate in formal and informal communities, which are the space required for socialisation [63]. 


\section{Externalisation:}

1) According to Chatti et al. [63], blogs support externalisation by offering a space for everyone to capture knowledge. They also enable immediate documentation of thoughts through discussions in different blogs. They are powerful for knowledge sharing in individualistic cultures. They can be suitable for sharing best practices and offers indirect communication, which can overcome the fear of assessment and criticisms. Moreover, blogs allow anonymous participation to overcome the barrier of structure in organisations. Blogs utilisation in organisations can result in long term orientation organisations, which focus on long term goals [53]. Blogs can be utilised to support diabetic citizens by sharing guidelines related to medication intake from healthcare professionals.

2) Wikis are also seen to be useful web tool for externalisation [63]. They facilitate the capturing of collective knowledge where every user can share her/his experience. This increases social interaction and empower collaborative knowledge capturing as knowledge in wikis can be created and modified by different individuals. Therefore, wikis are seen as helpful tool to overcome individualism, which is the national attribute that hinders externalisation. This knowledge can be in various forms such as spoken or written words, different formats of images, sound tracks such as music and lectures. Furthermore, contributions in wikis, which also can be anonymous, can be supported by top management in organisations [53]. This can overcome the of high power distance which occurs in cultures such as KSA. Wikis can be great mean to share appropriate exercises and medications best practices in written forms.

3) Besides its support to socialisation, social media can also support externalisation. Social media enable users to share and capture different knowledge about diabetes and scientific findings that may be presented in different formats such as text, images, videos or audios. For example, it can convert tips of diabetes medication dosage and tips into codified guidelines. Moreover, it can be facilitated through newly developed devices and software for knowledge sharing and capturing [63].

4) Question and answers page is another web tool that can support externalisation. It facilitates externalisation as it allows articulation and codifying of tacit knowledge. This process enables the conversion of knowledge from tacit form to explicit [64]. Questions and answers are useful way to externalise medical knowledge in forms of patient's questions and answers provided by healthcare professional. For example, best practice of how to measure blood glucose level.

\section{Combination:}

1) One of the effective web technologies for combination is document system. According to Natek [64], a document system allows participants to upload and download important documents of interests. It facilitates access to information. Nonaka and Takeuchi [4] indicated that open source to information is an important tool for combination. Moreover, uploading and downloading documents of interest ensures the practice of combination in Arabic culture, which per- 
forms combination only when the knowledge is considered to be valuable [43]. Document system can disseminate strategies of coexisting with diabetes mellitus and policies related to diabetes prevention and treatments.

2) Wikis are also seen to be effective tool for combination in the online community. Wikis build searchable and up to date learning content that can be distributed among different organisational boundaries [63]. In addition, wikis enable collaborative decision-making and decrease bureaucracy. This is prerequisite for combination as a consultative decision making is effective practice for this mode [4].

3) Blogs can support combination in a similar way to wikis. Blogs can provide valuable learning assists that are searchable and up to date and can be transferrable into social context [63]. Furthermore, they allow collaborative decision making and decrease bureaucracy. Blogs can share different strategies related to appropriate diet plan.

\section{Internalisation:}

1) $\mathrm{CoP}$ is one of the effective web tools for internalisation [64]. CoP can be provided for participants through social media where work-groups transfer knowledge among staffs who share the same interests or who are looking for a particular knowledge. To overcome cultural boundaries such as low uncertainty avoidance, rewarding valuable contributions will lead the organisation's community to value KS and increase it. Another way to reward contributions is by linking posts to the organisations network to value knowledge and give those contributors reputations as experts, which also overcomes individualism and then increase KS. CoP can be integrated with web forums by allowing participants of common interests, i.e. diabetic individuals, to share their experiences and their best practices [53]. Anonymous participation can be valuable in this type of web technology especially in high power distance cultures such as KSA. Another way to apply CoP is to provide a shared repository for information resources, which can be used to support diabetics in a more tailored way, according to gender, age or lifestyle [66]. Therefore, instructional videos can be valuable repository for knowledge that can implement internalisation through a CoP. Such and implementation can include sharing of guidelines related diet and exercise, which can be mastered by diabetics through the practice of "learning by doing".

2) Wikis are also an effective web tool for internalisation. Wikis enable individuals to contribute by sharing their experience in their speciality [53]. Wikis can be implemented as a CoP. They help individuals to capture knowledge of others and enable users to easily edit webpage contents [67]. Wikis can be adopted by top management in organisations to facilitate KS. Also anonymous participation is effective to encourage KM practice in high power distance cultures, such as KSA.

In summary, the application of the SECI model via web tools can facilitate knowledge dissemination practice effectively through overcoming a number of 
national and organisational hinderers of KM. This will play significant role in the control of diabetes non-health related complications in highly prevalent country of diabetes mellitus such as KSA. The socialisation mode can play significant role in overcoming these complications by enabling diabetic individuals to socialise with each other via the portal. The outcomes from such an action result in engaging diabetic individuals in group discussion about their complications and best practices. Diabetic citizens can also socialise and interact with healthcare professionals. In terms of externalisation, healthcare professionals can help patients overcome their complications can be overcome through converting useful guidelines, best practices and recommendations to comprehensible and appropriate media. The externalised recommendations can empower diabetic individuals to deal with different types of non-health related complications of diabetes mellitus such as identifying appropriate time and/or dosage of medication. Moreover, the externalisation mode can disseminate our tacit DM findings and make them explicit. This can benefit the community of diabetes mellitus by providing data in explicit form and make them available for further research and studies related to diabetes mellitus. The combination mode provides flexible way to integrate different aspects of guidelines and strategies for appropriate diabetic citizens taken into consideration their specific lifestyle, diet, medication issues and Ramadan requirements. In the internalisation mode, CoPs can be created where diabetic citizens are learning by doing and following different guidelines related to dealing with several barriers such as identifying the right physical activity and coexistence with the life style of diabetes mellitus.

\section{Conclusions}

The prevalence of diabetes mellitus in KSA requires serious attention to raise the awareness of diabetes self-management and education. The current paper shows that current practices are limited in KM with respect to the healthcare domain in the Kingdom. The integrated framework of $\mathrm{KM}$ and $\mathrm{KD}$, presented in this paper is an effective approach to support patients and healthcare professionals of diabetes mellitus in KSA. The SECI model is a valuable knowledge creation theory, which can address the factors hindering the practice $\mathrm{KM}$ and encourage diabetes self-management and education in the Kingdom through various technological means. The application of the SECI model can be supported by a number of web tools. These tools not only apply the four modes of the SECI model successfully, but they also have the ability to overcome a number of national and organizational factors that hinder knowledge dissemination in KSA. This will encourage KM practice in KSA in the domain of healthcare.

The current paper has two future research directions. The first future research direction is a DM study, which can reveal a number of non-health related complications of diabetes mellitus from a dataset of diabetic patients in KSA. The second future work opportunity includes development of a KM web portal to support diabetic patients to overcome the barriers extracted from the DM study. 
The portal can utilise different web tools to apply the four modes of the SECI model in order to share useful guidelines and best practices related to diabetes self-management and education effectively.

\section{References}

[1] Lorig, K.R., Sobel, D.S., Ritter, P.L., Laurent, D. and Hobbs, M. (2000) Effect of a Self-Management Program on Patients with Chronic Disease. Effective Clinical Practice, 4, 256-262.

[2] Ni, G., Wang, W., Wang, J., Zong, Z. and Xie, M. (2010) Research on the Knowledge Management System of the Vicarious Management Corporation. Information Science and Management Engineering, Xi'an, 7-8 August 2010, 62-67.

[3] McInerney, C.R. and Koenig, M.E.D. (2011) Knowledge Management (KM) Processes in Organizations: Theoretical Foundations and Practice. Synthesis Lectures on Information Concepts, Retrieval, and Services, 3, 1-96.

[4] Nonaka, I. and Takeuchi, H. (1995) The Knowledge-Creating Company: How Japanese Companies Create the Dynamics of Innovation. Oxford University Press.

[5] Aljumah, A.A., Ahamad, M.G. and Siddiqui, M.K. (2013) Application of Data Mining: Diabetes Health Care in Young and Old Patients. The Journal of King Saud University Computer and Information Sciences, 25, 127-136.

[6] Esfandiari, N., Babavalian, M.R., Moghadam, A.M.E. and Tabar, V.K. (2014) Knowledge Discovery in Medicine: Current Issue and Future Trend. Expert Systems with Applications, 41, 4434-4463.

[7] Dalkir, K. and Liebowitz, J. (2011) Knowledge Management in Theory and Practice. MIT Press.

[8] Cao, J., Yao, Z., Li, Y., Zhai, C. and Xu, B. (2010) Utilizing SECI Model for Knowledge Management in Library. International Conference on Educational and Information Technology, Vol. 3, 62-64.

[9] Chikh, A. (2011) A Knowledge Management Framework in Software Requirements Engineering Based on the SECI Model. Journal of Software Engineering and Applications, 4, 718-728. https://doi.org/10.4236/jsea.2011.412084

[10] Li, Y.-H., Huang, J.-W. and Tsai, M.-T. (2009) Entrepreneurial Orientation and Firm Performance: The Role of Knowledge Creation Process. Industrial Marketing Management, 38, 440-449.

[11] Alshammari, T.M. (2016) Patient's Medicinal Knowledge in Saudi Arabia: Are We Doing Well? Saudi Pharmaceutical Journal, 24, 560-562.

[12] Szulanski, G. (2001) Knowledge Creation: A Source of Value. Academy of Management Review, 26, 318-320. https://doi.org/10.2307/259128

[13] Scott, J.E. (1998) Organizational Knowledge and the Intranet. Decision Support Systems, 23, 3-17.

[14] Ziebland, S., Lavie-Ajayi, M. and Lucius-Hoene, G. (2014) The Role of the Internet for People with Chronic Pain: Examples from the DIPEx International Project. British Journal of Pain, 9, 62-64. https://doi.org/10.1177/2049463714555438

[15] American Diabetes Association (2013) Diagnosis and Classification of Diabetes Mellitus. Diabetes Care, 36, 67-74.

[16] Ministry of Health (2016) Health Days 2016-World Diabetes Day. http://www.moh.gov.sa/en/HealthAwareness/healthDay/2016/Pages/HealthDay-201 
6-11-14.aspx

[17] Zhang, P., Zhang, X., Brown, J., Vistisen, D., Sicree, R., Shaw, J. and Nichols, G. (2010) Global Healthcare Expenditure on Diabetes for 2010 and 2030. Diabetes Research and Clinical Practice, 87, 293-301.

[18] Shaw, J.E., Sicree, R.A. and Zimmet, P.Z. (2010) Global Estimates of the Prevalence of Diabetes for 2010 and 2030. Diabetes Research and Clinical Practice, 87, 4-14.

[19] Ministry of Health (2011) Health Statistical Year Book 1432H. http://www.cdsi.gov.sa/english/

[20] Ministry of Health (2014) Statistical Year Book 1435H. http://www.moh.gov.sa/en/Ministry/Statistics/Book/Pages/default.aspx

[21] International Diabetes Federation (2014) Saudi Arabia International Diabetes Federation. http://www.idf.org/membership/mena/saudi-arabia

[22] International Diabetes Federation (2015) IDF Diabetes Atlas.

[23] Centers for Disease Control and Prevention (2016) Global Health-Saudi Arabia. https://www.cdc.gov/globalhealth/countries/saudi arabia/

[24] Péres, D.S., dos Santos, M.A., Zanetti, M.L. and Ferronato, A.A. (2007) Difficulties of Diabetic Patients in the Illness Control: Feelings and Behaviors. Revista Latino-Americana de Enfermagem, 15, 1105-1112. https://doi.org/10.1590/S0104-11692007000600008

[25] Blonde, L. (2005) Current Challenges in Diabetes Management. Clinical Cornerstone, 7.

[26] Onwudiwe, N.C., Mullins, C.D., Winston, R.A., Shaya, F.T., Pradel, F.G., Laird, A. and Saunders, E. (2014) Barriers to Self-Management of Diabetes: A Qualitative Study among Low-Income Minority Diabetics. South African Journal of Diabetes and Vascular Disease, 11, 61-65.

[27] Sweileh, W.M., Zyoud, S.H., Abu Naba, R.J., Deleq, M.I., Enaia, M.I., Nassar, S.M. and Al-Jabi, S.W. (2014) Influence of Patients? Disease Knowledge and Beliefs about Medicines on Medication Adherence: Findings from a Cross-Sectional Survey among Patients with Type 2 Diabetes Mellitus in Palestine. BMC Public Health, 14, 94. https://doi.org/10.1186/1471-2458-14-94

[28] Zargar, A. (2017) Diabetes Control during Ramadan Fasting. Cleveland Clinic Journal of Medicine, 84, 352-356. https://doi.org/10.3949/ccjm.84a.16073

[29] Hassanein, M., Al-Arouj, M., Hamdy, O., Bebakar, W.M.W., Jabbar, A., Al-Madani, A., Hanif, W., Lessan, N., Basit, A., Tayeb, K., Omar, M., Abdallah, K., Al Twaim, A., Buyukbese, M.A., El-Sayed, A.A. and Ben-Nakhi, A. (2017) Diabetes and Ramadan: Practical Guidelines. Diabetes Research and Clinical Practice, 126, 303-316.

[30] Powers, M.A., Bardsley, J., Cypress, M., Duker, P., Funnell, M.M., Fischl, A.H., Maryniuk, M.D., Siminerio, L. and Vivian, E. (2017) Diabetes Self-Management Education and Support in Type 2 Diabetes. The Diabetes Educator, 43, 40-53. https://doi.org/10.1177/0145721716689694

[31] Deakin, T., McShane, C.E., Cade, J.E. and Williams, R.D.R.R. (2005) Group Based Training for Self-Management Strategies in People with Type 2 Diabetes Mellitus. The Cochrane Database of Systematic Reviews, 2, CD003417. https://doi.org/10.1002/14651858.CD003417.pub2

[32] Herbig, B., Bussing, A. and Ewert, T. (2001) The Role of Tacit Knowledge in the Work Context of Nursing. Journal of Advanced Nursing, 34, 687-695. https://doi.org/10.1046/j.1365-2648.2001.01798.x 
[33] Thornton, T. (2006) Tacit Knowledge as the Unifying Factor in Evidence Based Medicine and Clinical Judgement. Philosophy, Ethics, and Humanities in Medicine, 1, 2. https://doi.org/10.1186/1747-5341-1-2

[34] Kothari, A., Rudman, D., Dobbins, M., Rouse, M., Sibbald, S. and Edwards, N. (2012) The Use of Tacit and Explicit Knowledge in Public Health: A Qualitative Study. Implementation Science, 7, 20. https://doi.org/10.1186/1748-5908-7-20

[35] Boateng, W. (2008) Knowledge Management in Evidence-Based Medical Practice: Does the Patient Matter? Electronic Journal of Knowledge Management, 8, 281-292.

[36] Hovlid, E., Bukve, O., Haug, K., Aslaksen, A.B. and von Plessen, C. (2012) Sustainability of Healthcare Improvement: What Can We Learn from Learning Theory? BMC Health Services Research, 12, 235. https://doi.org/10.1186/1472-6963-12-235

[37] Wickramasinghe, N. and Davison, G. (2004) Making Explicit the Implicit Knowledge Assets in Healthcare: The Case of Multidisciplinary Teams in Care and Cure Environments. Health Care Management Science, 7, 185-195. https://doi.org/10.1023/B:HCMS.0000039381.02400.49

[38] Al-Ghamdi, S.A., Mahmoud, M.A., Alammari, M.A., Al Bekairy, A.M., Alwhaibi, M., Mayet, A.Y. and Aljadhey, H.S. (2012) The Outcome of Pharmacist Counseling at the Time of Hospital Discharge: An Observational Nonrandomized Study. Annals of Saudi Medicine, 32, 492-497. https://doi.org/10.5144/0256-4947.2012.492

[39] Alkatheri, A. and Albekairy, A. (2013) Does the Patients' Educational Level and Previous Counseling Affect Their Medication Knowledge? Annals of Thoracic Medicine, 8, 105. https://doi.org/10.4103/1817-1737.109823

[40] Ridings, C., Gefen, D. and Arinze, B. (2002) Some Antecedents and Effects of Trust in Virtual Communities. The Journal of Strategic Information Systems, 11, 271-295.

[41] Al-Alawi, A.I., Al-Marzooqi, N.Y. and Mohammed, Y.F. (2007) Organizational Culture and Knowledge Sharing: Critical Success Factors. Journal of Knowledge Management, 11, 22-42. https://doi.org/10.1108/13673270710738898

[42] Hutchings, K. and Michailova, S. (2003) Facilitating Knowledge Sharing in Russian and Chinese Subsidiaries: The Importance of Groups and Personal Networks.

[43] Weir, D. and Hutchings, K. (2005) Cultural Embeddedness and Contextual Constraints: Knowledge Sharing in Chinese and Arab Cultures. Knowledge and Process Management, 12, 89-98. https://doi.org/10.1002/kpm.222

[44] Gold, A.H., Malhotra, A. and Segars, A.H. (2001) Knowledge Management: An Organizational Capabilities Perspective. Journal of Management Information Systems, 18, 185-214. https://doi.org/10.1080/07421222.2001.11045669

[45] Alavi, M., Kayworth, T.R. and Leidner, D.E. (2015) An Empirical Examination of the of Organizational Influence Culture on Knowledge Practices Management. Journal of Management Information Systems, 22, 191-224.

[46] Bradley, M. (2000) Working Knowledge: How Organizations Manage What They Know. New Library World, Emerald Group Publishing Limited, 282-287.

[47] Schein, E.H. (2004) Organizational Culture and Leadership. 3rd Edition.

[48] Park, H., Ribière, V. and Schulte, W.D. (2004) Critical Attributes of Organizational Culture That Promote Knowledge Management Technology Implementation Success. Journal of Knowledge Management, 8, 106-117. https://doi.org/10.1108/13673270410541079

[49] Mowery, B. (2015) Report Information from ProQuest.

[50] Albrithen, A. and Yalli, N. (2015) Medical Social Workers' Perceptions Related to 
Interprofessional Teamwork at Hospitals. Journal of Social Service Research, 41, 722-731. https://doi.org/10.1080/01488376.2015.1068723

[51] ALHussain, A.Z. (2011) Barriers to Knowledge Management in Saudi Arabia.

[52] Glisby, M. and Holden, N. (2003) Contextual Constraints in Knowledge Management Theory: The Cultural Embeddedness of Nonaka's Knowledge-Creating Company. Knowledge and Process Management, 10, 29-36. https://doi.org/10.1002/kpm.158

[53] Ray, D. (2014) Overcoming Cross-Cultural Barriers to Knowledge Management Using Social Media. Journal of Enterprise Information Management, 27, 45-55. https://doi.org/10.1108/JEIM-09-2012-0053

[54] Sabri, H. (2005) Knowledge Management in Its Context: Adapting Structure to a Knowledge Creating Culture. International Journal of Commerce and Management, 15, 113-128.

[55] Nazari, J.A., Herremans, I.M., Isaac, R.G., Manassian, A. and Kline, T.J. (2009) Organizational Characteristics Fostering Intellectual Capital in Canada and the Middle East. Journal of Intellectual Capital, 10, 135-148. https://doi.org/10.1108/14691930910922950

[56] Hofstede, G. (2003) Culture's Consequences: Comparing Values, Behaviors, Institutions, and Organizations across Nations. Behaviour Research and Therapy, 41, 861862.

[57] Hofstede, G. (2001) Culture's Consequences: Comparing Values, Behaviors, Institutions and Organizations across Nations. SAGE, Thousand Oaks, London, New Delhi.

[58] Nonaka, I., Toyama, R. and Konno, N. (2000) SECI, Ba and Leadership: A Unified Model of Dynamic Knowledge Creation. Long Range Planning, 33, 5-34.

[59] Nonaka, I. (1994) A Dynamic Theory of Organizational Knowledge Creation. Organization Science, 5, 14-37. https://doi.org/10.1287/orsc.5.1.14

[60] Nonaka, I. and Konno, N. (1998) The Concept of Ba: Building a Foundation for Knowledge Creation. California Management Review, 40, 40-54. https://doi.org/10.2307/41165942

[61] Almuayqil, S., Atkins, A.S. and Sharp, B. (2015) Integrated Framework of Knowledge Management and Knowledge Discovery to Support E-Health for Saudi Diabetic Patients. International Journal of Advanced Life Sciences, 7, 111-121.

[62] Almuayqil, S., Atkins, A.S. and Sharp, B. (2016) Ranking of E-Health Barriers Faced by Saudi Arabian Citizens, Healthcare Professionals and IT Specialists in Saudi Arabia. Health, 1004-1013. https://doi.org/10.4236/health.2016.810104

[63] Chatti, M.A., Klamma, R., Jarke, M. and Naeve, A. (2007) The Web 2.0 Driven SECI Model Based Learning Process. Advanced Learning Technologies, Niigata, 18-20 July 2007, Vol. 5, 780-782. https://doi.org/10.1109/ICALT.2007.256

[64] Natek, S. (2016) Knowledge Management Systems Support Seci Model of Knowledge-Creating Process Abstract.

[65] Constantinides, E. and Fountain, S.J. (2008) Special Issue Papers Web 2.0: Conceptual Foundations and Marketing Issues. Journal of Direct, 9, 231-244.

[66] Hoadley, C. (2012) What Is a Community of Practice and How Can We Support It? In: Theoretical Foundations of Learning Environments, 286.

[67] Dickson, D. (2009) Using Wiki Technology to Create Communities of Practice. Rochester Institute of Technology, Rochester. 
Submit or recommend next manuscript to SCIRP and we will provide best service for you:

Accepting pre-submission inquiries through Email, Facebook, LinkedIn, Twitter, etc. A wide selection of journals (inclusive of 9 subjects, more than 200 journals)

Providing 24-hour high-quality service

User-friendly online submission system

Fair and swift peer-review system

Efficient typesetting and proofreading procedure

Display of the result of downloads and visits, as well as the number of cited articles Maximum dissemination of your research work

Submit your manuscript at: http://papersubmission.scirp.org/

Or contact iim@scirp.org 